\title{
Principal processes occurring at simultaneous bombardment of tungsten by carbon and deuterium ions
}

\author{
Ivan Bizyukov \\ Kharkiv National University, Faculty of Physics and Technologies, 31 Kurchatov Ave., \\ Kharkiv 61108, Ukraine
}

\author{
Karl Krieger \\ Max-Planck-Institut für Plasmaphysik, EURATOM Association, Boltzmannstr. 2, \\ 85748 Garching, Germany
}

\begin{abstract}
Simultaneous bombardment of tungsten with deuterium and carbon leads to both tungsten erosion and implantation of carbon and deuterium in the $\mathrm{W}$ surface. These processes have been studied experimentally by simultaneous bombardment of magnetron deposited W layers with $\mathrm{D}$ and $\mathrm{C}$ ions at energies in the $\mathrm{keV}$ range. The experimental results were compared to numerical simulations using the TRIDYN code. The key parameter separating steady state $\mathrm{W}$ erosion from continuous growth of a $\mathrm{C}$ layer on the surface is the $\mathrm{C}$ fraction in the incident flux. The dynamical change of the surface composition with increasing fluence was monitored in-situ by ion beam analysis. The experimental results are well reproduced by the TRIDYN simulations, which are based on a kinematic model of ion scattering in the target. Significant contributions by chemical effects outside the scope of the TRIDYN model can therefore be excluded. A negligible contribution of chemical effects is also supported by the measured concentrations of retained D, which are not sufficient to create a significant contribution of chemical reactions with carbon to implantation and sputtering. Surface roughness of the specimens was found to be the key parameter for the observed shift of the transition point from continuous C layer growth to steady state tungsten erosion with increasing D fraction in the incident ions.
\end{abstract}


Keywords: simultaneous ion bombardment, carbon implantation, deuterium depth distribution, tungsten sputtering, transition point.

\section{Introduction}

In the design of the International Thermonuclear Experimental Reactor (ITER), tungsten (W) will be used for plasma-facing components (PFCs) in the divertor baffle region, which are exposed to high particle and heat fluxes [1]. Experience with W PFCs in ASDEX Upgrade has shown that tungsten is sputtered mainly by multiply charged low-Z impurities accelerated in the sheath potential [2]. Sputtering by gaseous impurity ions, like O, Ar or Ne is already well understood and can be predicted with good accuracy by existing models. However, more complicated sputtering and deposition effects occur for carbon (C) and beryllium $(\mathrm{Be})$, because this impurities are non-volatile and will be implanted in the $\mathrm{W}$ surface and may also form deposited layers covering the original surface. In ITER carbon target plates are directly adjacent to the tungsten baffle areas and therefore, $\mathrm{C}$ will likely be together with beryllium the dominant impurity species in the incident fuel ion flux at the W PFCs $[3,4]$.

At room temperature, bombardment of a tungsten surface with only carbon ions at normal incidence angle results in complete coverage of the $\mathrm{W}$ surface with a $\mathrm{C}$ layer after a certain irradiation fluence thus preventing further $\mathrm{W}$ sputtering $[5,6]$. This process is well understood and can be well reproduced with codes based on the binary-collision approximation, like the Monte-Carlo code TRIDYN [7].

The more complicated case of simultaneous impact of D and C ions on a W surface may lead to one of the following scenarios, studied in $[8,9]$. In the first scenario, the $\mathrm{C}$ fraction in the incident flux $f_{C}$ is sufficiently low that implantation of $\mathrm{C}$ is always balanced by $\mathrm{C}$ removal due to $\mathrm{D}$ ion sputtering and therefore prevent formation of a $\mathrm{C}$ layer, exposing the $\mathrm{W}$ surface 
to continuous sputtering by the incident $\mathrm{C}$ and $\mathrm{D}$ ions. In the second scenario, $f_{C}$ is so high that eventually implantation of $\mathrm{C}$ prevails over its re-erosion, leading to formation of a $\mathrm{C}$ layer on top of the $\mathrm{W}$ bulk material. At normal incidence and at ion energies in the $\mathrm{keV}$ range and below, the critical carbon fraction, $f_{C}$, defining the transition between the two principal scenarios is well below $25 \%$. However, single ion beam experiments using $\mathrm{CH}_{3}$ ions are restricted to a value of $f_{C}=25 \%$. Therefore, to determine the key parameter $f_{C}$ it is necessary to use an experimental setup where the carbon fraction among the incident ions can be varied.

At simultaneous bombardment, the surface composition will be subject to the competition between erosion of the surface, deposition and implantation of carbon and deuterium. At the same time the surface composition itself influences the sputtering rates of the elements contributing to the mixed surface material. The non-linear interdependency between the composition of the mixed surface and sputtering can be well reproduced by TRIDYN in cases, where kinematic processes dominate [10]. However, simultaneous bombardment with $\mathrm{D}$ and $\mathrm{C}$ ions will result in sputtering of implanted $\mathrm{C}$ due to both kinematics and chemical processes with unknown partial contributions of each mechanism.

Disagreement between TRIDYN simulation and experiment was already observed at $f_{C}$ $=25 \%\left(\mathrm{E}_{\mathrm{C}}=2.4 \mathrm{keV}, \mathrm{E}_{\mathrm{H}}=0.2 \mathrm{keV}\right.$, normal incidence angle, polished poly-crystalline $\left.\mathrm{W}\right)$ and reported in [8]. The purely kinematic model was extended by applying additional sputtering of $\mathrm{C}$ due to chemical effects [9] which allowed to model the decreased $\mathrm{C}$ layer growth compared to pure $\mathrm{C}$ bombardment. However, the experiments described in $[8,9]$ were restricted to a $25 \%$ fraction of $\mathrm{C}$ in the incident flux. Furthermore, the reported results were obtained by weight change measurements, which can be significantly affected by a nonuniform lateral distribution of the ion current. Therefore, the contribution of chemical effects in addition to sputtering of implanted carbon atoms by $\mathrm{D}$ and $\mathrm{C}$ ions was still uncertain.

To quantify the influence of surface roughness and of chemical effects on W sputtering 


\section{Page 4}

and $\mathrm{C}$ implantation, tungsten was bombarded simultaneously with $\mathrm{D}$ and $\mathrm{C}$ ion beams with varying carbon fractions $f_{C}$ at room temperature. In the simulations surface roughness is taken into account by replacing the macroscopic surface incidence angle with an effective incidence angle for the surface, which can be interpreted as an average over the microscopic surface morphology [6]. Chemical sputtering of carbon is simulated by an appropriate decrease of the surface binding energy of $\mathrm{C}$ atoms.

\section{Experimental}

The experiments were performed with mass-separated $12 \mathrm{keV} \mathrm{C}_{2}$ and $9 \mathrm{keV} \mathrm{D}_{3}$ ions with energies chosen to obtain maximum beam flux densities. These energies are significantly higher than typically expected at the ITER baffle regions [3]. However, it should be noted that the objective of these experiments is the model validation of numerical simulations, which can then in turn be used to predict the erosion caused by physical sputtering at typical ITER parameters. This approach is justified because the simulations use the $\mathrm{C}-\mathrm{Kr}$ interaction potential [11] in the entire energy range of both the dual beam experiment and ITER.

Apart from the projectile energies the target temperature is a key parameter, which determines various carbon related processes like diffusion, chemical erosion and radiationenhanced sublimation (RES). In the experiments of this study all bombarded samples were kept at room temperature during the experiments and consequently one can neglect diffusion [12] and RES [9].

For the interpretation of the ion beam bombardment processes it is assumed that each $\mathrm{C}$ projectile atom has fractional incidence energy of $6 \mathrm{keV}$ and each $\mathrm{D}$ projectile has fractional incidence energy of $3 \mathrm{keV}$. The focal points of both ion beams were set behind the bombarded surface to achieve optimally uniform bombardment over the analyzed area. This also provided a good accuracy of fluence measurements, assuming a Gaussian ion beam shape with a 


\section{Page 5}

diameter of $4 \mathrm{~mm}$. The angle of incidence for both species was $15^{\circ}$. Ion beam analysis (IBA) with $2.5 \mathrm{MeV}{ }^{3} \mathrm{He}$ ions and $2 \mathrm{MeV}{ }^{4} \mathrm{He}$ was performed in-situ between the bombardment sessions, monitoring the evolution of the surface composition with increasing fluence. The use of W layers as a bombarded sample allows to use Rutherford back-scattering (RBS) for the measurement of the $\mathrm{W}$ areal density with an accuracy of $1 \%$. The change of the $\mathrm{W}$ areal density is calculated as the difference between the initial and the post-bombardment values. In principle the main error source is the uncertainty of the stopping power, which, however, cancels out because the decrease of the areal density is measured relatively to the initial value.

For the validation of simulations, a key point of the experiment is the ability to detect small amounts of implanted C by Nuclear Reaction Analysis (NRA). Carbon was quantified using the nuclear reaction ${ }^{12} \mathrm{C}\left({ }^{3} \mathrm{He}, \mathrm{p}\right){ }^{14} \mathrm{~N}$ [13]. The measurements were evaluated using a reference a-C:D layer sample with known $\mathrm{D}$ and $\mathrm{C}$ areal densities with a resulting accuracy of $<10 \%$. Depth profiles of implanted D were measured using the nuclear reaction $\mathrm{D}\left({ }^{3} \mathrm{He}, \mathrm{p}\right) \alpha$ and detecting the $\alpha$-particles at shallow exit angle (scattering angle $105^{\circ}$ ). Further details of the experimental setup, as well as of the measurement technique are described in [14].

Target samples were prepared by magnetron sputter deposition of $\mathrm{W}$ layers (thickness $\approx 280 \pm 30 \mathrm{~nm}$ ) onto pyrolitic graphite with an intermediate $\mathrm{Cu}$ layer (thickness $\approx 380 \pm 40 \mathrm{~nm}$ ) applied before in the same process. The low-Z impurity content of the layers was measured by $\mathrm{X}$-ray photoelectron spectroscopy $(<1 \%$ for $\mathrm{C}$ and $\mathrm{N}$ and $<10 \%$ for $\mathrm{O})$. The thickness of the W layer was chosen to be large enough to prevent any interaction of the projectiles and recoils with the layers below. Further details on structure and properties of the W layers can be found in [15]. The mean projectile ranges of $6 \mathrm{keV} \mathrm{C}$ and $3 \mathrm{keV} \mathrm{D}$ are $\mathrm{R}_{\mathrm{C}} \approx 10 \mathrm{~nm}$ and $\mathrm{R}_{\mathrm{D}} \approx 20 \mathrm{~nm}$ respectively, for a pure $\mathrm{W}$ surface. The average roughness of the $\mathrm{W}$ layer was determined by atomic force microscopy of a square area of $5 \times 5 \mu \mathrm{m}$ to a value of $\mathrm{R}_{\mathrm{a}} \approx 200-300 \mathrm{~nm}$. This is much larger than the projectile ranges of the ions. Since $R_{a}>>R_{C}, R_{D}$, surface roughness has 
to be taken into account.

\section{Surface modification due to $D$ bombardment}

In the binary collision model, used as base of codes like TRIDYN, D ions contribute only to collisional processes. Other effects, like blistering or creation of chemical bonds are outside the scope of this model. One must expect that the influence of D bombardment on a surface can not be described by collision effects only. The accuracy and quality of TRIDYN predictions will therefore critically depend on the relative importance of these additional factors, which are discussed in the subsequent sub-sections.

\subsection{Blistering}

Blistering of metal surfaces under high fluence bombardment with hydrogen ion beams is a well known effect [16]. Blistering of a W surface was observed both after bombardment with a single D ion beam [17] and after bombardment with a mixed D-C ion beam [18]. If the bulk surface is replaced by tungsten films, the blistering effect will be observed usually at lower fluence. An example of such blisters is shown in Figure 1(a) for a target bombarded by a single D ion beam. Similar blisters are obtained also by simultaneous D and C ion beam bombardment. At the samples used in this study, blistering was, however, not a well reproducible effect. Post bombardment studies of some sample surfaces showed no blistering in spite of the same irradiation fluence.

Since the sample has a multilayer structure, one has to clarify the location of the blisters. A dedicated sample with a W-Cu multilayer deposited onto a Si single crystal polished wafer, was irradiated with a D ion beam applying a fluence, high enough to produce blisters and then cracked along the crystal axis. An image of the cross-section was obtained with scanning electron microscopy as shown in Figure 1(b). One can see that the shape of the blister cup results from exfoliation of the $\mathrm{W}$ layer, while the $\mathrm{Cu}$ layer and the substrate are 


\section{Page 7}

unaltered. The deformation of the $\mathrm{W}$ layer also results in a non-flat surface, which will influence sputtering and implantation processes similar to the presence of roughness. For the samples deposited onto polished pyrolitic graphite the roughness of the substrate was already approximately the same as the roughness of the blistering surface. Thus, the approximation for the simulation of ion interaction with rough surface suggested in [6] is also applicable for the given projectile-target system. Because the presence of blisters in this case does not further alter the surface morphology and the blister voids are beyond the range of the incident ions, they do not need to be taken into account in the modeling.

\section{2. $D$ retention in mixed $W-C-D$ surfaces}

Retention of the implanted $\mathrm{D}$ in the implantation zone results in contributions of $\mathrm{D}$ recoils in the collision cascades of incident projectile ions. Codes based on the binary collision approximation do usually not provide a correct description of the distribution of the implanted gaseous atoms, because they do not include the diffusion and trapping of $\mathrm{D}$. The density of implanted D in W is usually low, even in the implantation zone it is typically below $10 \%$ [19]. However, simultaneous implantation with $\mathrm{C}$ can dramatically increase the number of traps in a mixed material surface and increase the $\mathrm{D}$ retention in the implantation zone significantly above this value.

For both the case of pure D bombardment and of simultaneous D-C bombardment the resulting D depth profile in the $\mathrm{W}$ layers was measured with NRA. Figure 2(a) shows the measured D depth profile for pure D bombardment after a total fluence of $15 \times 10^{18} \mathrm{~cm}^{-2}$. Figure 2(b) shows the measured depth profiles of both D and C for simultaneous D and C bombardment with $f_{C}=6 \%$ after a total fluence of $4.1 \times 10^{18} \mathrm{~cm}^{-2}$. According to previous studies, the limited film depth provides a limited number of traps, so that the D areal density increases only by $\approx 15 \%$ when increasing the fluence from $4 \times 10^{18} \mathrm{~cm}^{-2}$ to $15 \times 10^{18} \mathrm{~cm}^{-2}$ [15]. Therefore it is justified to compare the D depth profile of the case of pure D bombardment to 


\section{Page 8}

that obtained with simultaneous $\mathrm{D}$ and $\mathrm{C}$ bombardment at a lower fluence value. The comparison of both D profiles shows that in the given fluence range the mixed surface does not increase the $\mathrm{D}$ retention. Moreover, the typical concentration of $\mathrm{D}$ in the implantation zone remains below $10 \%$ despite the concentration of the $\mathrm{C}$ atoms in the same depth range is a factor of 10 higher than that of the $\mathrm{D}$ atoms. Therefore, the effect of the $\mathrm{D}$ retention is low enough to be neglected in calculations.

\subsection{Chemical sputtering of implanted $C$}

Kinetically induced chemical sputtering of carbon by ions of hydrogen isotopes at room temperature is a well known process [20]. The recoils of the collisional cascade from projectile ions break $\mathrm{C}-\mathrm{C}$ bonds and atoms of retained hydrogen isotopes in turn may attach to the free bonds. If the resulting hydrocarbons reach the surface they have a lower surface binding energy $E_{S B E}$ than $C$ atoms. Typically reported values of $E_{S B E}$ for hydrocarbons scatter from $E_{S B E}=1 \mathrm{eV}[21,22]$ to $E_{S B E}=2.8-4.5 \mathrm{eV}[23,24]$. The chemical sputtering yield peaks at an ion energy of several hundreds of $\mathrm{eV}$ and practically disappears at several $\mathrm{keV}$ due to domination of high energy recoils near the surface.

In case of mixed W-C-D surfaces, kinetically induced chemical sputtering of the hydrocarbons may exist even at the energies of $\mathrm{C}$ and $\mathrm{D}$ ions in the discussed simultaneous bombardment experiments. Since the mass difference between projectiles and $\mathrm{W}$ atoms is at least one order of magnitude, low energy W recoils may only transfer a small fraction of the energy of incident particles to $\mathrm{C}$ atoms.

The maximal energy, delivered to an hydrogenated $\mathrm{C}$ atom on the surface by the simplest scattering case involving at least one $\mathrm{W}$ atom, i.e. through the channel $(3 \mathrm{keV}$ $\mathrm{D}$ ion $) \rightarrow(\mathrm{W}$ recoil $) \rightarrow(\mathrm{C})$, is only $\approx 30 \mathrm{eV}$. Similarly, the channel $(\mathrm{C}$ ion $) \rightarrow(\mathrm{W}$ recoil $) \rightarrow(\mathrm{C})$ allows to deliver $\approx 317 \mathrm{eV}$. Therefore the energy transfer is in a range where kinetically induced chemical sputtering may contribute significantly to $C$ erosion at the surface. This can 


\section{Page 9}

be approximated using the model for physical sputtering modified by a lower surface binding energy [20]. Using the data obtained by comparing experimental results with those of TRIM.SP simulations [23, 24], one obtains an estimate of the surface binding energy of $C$ to C atoms of $E_{S B E}=3.6 \mathrm{eV}$. It should be noted that in the simulations the unknown surface binding energy of $\mathrm{C}$ to $\mathrm{W}$ atoms on the surface has been approximated by the average of the surface binding energies of $\mathrm{C}$ to $\mathrm{C}$ and $\mathrm{W}$ to $\mathrm{W}$ respectively. The resulting $E_{S B E}$ value can therefore exceed the value $3.6 \mathrm{eV}$ for pure carbon with increasing $\mathrm{W}$ concentration on the surface.

Figure 3 shows the influence of the surface binding energy on the calculated depth profile of $\mathrm{C}$ implanted simultaneously with $\mathrm{D}$ ions. At lower surface binding energy, the $\mathrm{C}$ concentration is lower by a factor of two at a depth of 1-2 $\mathrm{nm}$, because low energy $\mathrm{C}$ recoils created near the surface are capable to reach the surface and escape. This process is dependent on the $\mathrm{C}$ concentration on the surface, i.e. at higher $\mathrm{C}$ concentration $\mathrm{C}$ recoils with lower energy will be able to escape the solid. One should note that the low energy sputtered $\mathrm{C}$ atoms originating from the first 1-2 $\mathrm{nm}$ below the surface mainly affect the near surface $\mathrm{C}$ concentration, while the $\mathrm{C}$ concentration at higher depth ranges is only a result of the balance between sputtering and implantation at steady-state. Because of the limited depth resolution, the near surface effects are not visible in the experimental depth profile (Figure $2 b$ ).

A similar approach to the simulation of chemical sputtering of $\mathrm{C}$ implanted into $\mathrm{W}$ was used in [9], but chemical sputtering was simulated by deleting a certain amount of $\mathrm{C}$ atoms per fluence step from the top few nm according to previous calibration experiments.

As chemically sputtered $\mathrm{C}$ atoms are assumed to be hydrogenated leading to the assumed lower surface binding energy used in the simulation, chemical sputtering depends critically on the availability of D atoms in the near surface layers. However, it has been shown in subsection 3.2 that the D concentration in the implantation zone is significantly lower in 
comparison to the $\mathrm{C}$ concentration. The comparatively low amount of $\mathrm{D}$ should not be enough to produce a significant contribution to the sputtering of $\mathrm{C}$ atoms from the surface in comparison to physical sputtering. Therefore, kinetically induced chemical sputtering is not expected to significantly contribute to the experimental results.

\section{Parametric evolution of surface composition}

Simultaneous bombardment of $\mathrm{W}$ surfaces with gaseous and volatile ions leads to one of two scenarios depending on parameters of the incident flux: either steady-state surface erosion or continuous growth of a $\mathrm{C}$ layer [10]. The initial phase of both scenarios is characterized by a dynamical change of the $\mathrm{W}$ and $\mathrm{C}$ concentration in the implantation region with fluence. Figure 4 shows the parametric representation of the evolution of the elemental surface composition using implantation-sputtering curves introduced in [6]. Using this approach, the explicit dependence of the experimental results on fluence cancels out. This has the advantage that experimental errors in the determination of the local fluence due to the inhomogeneous distribution of the ion beam flux over the surface do not affect the results. Implantation-sputtering curves have been simulated with TRIDYN taking into account roughness effects by using a nominal angle of incidence $\alpha=38^{\circ}$, which is larger than the actual macroscopic angle of ion incidence $\alpha=15^{\circ}$. The nominal value of the incidence angle has been obtained by fitting a number of experimental results with various values of $f_{C}$. Figure 4(a) shows typical implantation sputtering curves corresponding to steady-state erosion of the surface. Initially, $\mathrm{W}$ sputtering is accompanied by $\mathrm{C}$ implantation. The latter is increased until the amount of $\mathrm{C}$ in the surface reaches a steady-state value where implantation is balanced by sputtering with D and C ions. Experimental and theoretical data show that the steady-state value of the $\mathrm{C}$ areal density increases with $f_{C}$. At the same time, tungsten is continuously eroded, measured by a continuous decrease of the $\mathrm{W}$ areal density in the target 
Page 11

layer with increasing fluence. The experimental data for $f_{C}=9 \%$ differs from the other two cases by the absence of blistering. However, the observed qualitative agreement in case of the blistered surfaces is as good as in the case of the intact layer. This is expected because as discussed in section 3, the blister voids are below the range of the ion projectiles and the blisters do not significantly alter the morphology of the already relatively rough surface of the samples. The dynamical change of the surface at low fluences shows significant deviations between experimental data and simulations as seen in Figure 4(a). This indicates that the approximation of the surface roughness effects by using a single nominal angle of incidence is not sufficient to describe the influence of the surface morphology on sputtering and implantation.

Typical evolutions of surface composition for the second scenario, where the W surface is finally covered by a C layer, are shown in Figure 4(b). At the maximum accessible fluence in these experiments, complete coverage of the W surface has not yet occurred. Theoretically, the required fluence to reach complete coverage diverges towards the transition point from $\mathrm{W}$ sputtering to $\mathrm{C}$ layer growth. The transition to the $\mathrm{C}$ layer growth scenario in the presented data of Figure 4(b) is inferred from the monotonic increase of the $\mathrm{C}$ areal density over the predictions of the simulation. One should note that the chosen nominal incidence angle well describes the sputtering of the surface, but the simulation using this value does not predict the formation of a $\mathrm{C}$ layer. The transition point to continuous $\mathrm{C}$ layer growth occurs in the simulations at a higher $f_{C}$ value than indicated by the experimental data.

\section{Ion-surface interactions at steady-state $W$ erosion}

While the initial evolution of the surface composition is rather complicated, the scenario of steady-state $\mathrm{W}$ erosion can be well described by just two experimental parameters. The first parameter is the areal density of implanted $\mathrm{C}$, which is constant at steady state. As the C 
areal density is given by $f_{C}$ in the incident beams, which itself is approximately constant across the bombarded area; the steady state value $\mathrm{C}$ areal density is also not affected by errors in the fluence measurement. The second parameter is the sputtering yield of $\mathrm{W}$ atoms, $Y_{W}$, which can be determined with an experimental error given mainly by the limited accuracy of the fluence measurement compared to the much smaller error of the ion beam analysis. The experimental methods for the measurement of these parameters are described in detail in [10].

A numerical study of these parameters as a function of $f_{C}$ was performed using the following combinations of assumptions in TRIDYN:

- Default TRIDYN model without additional assumptions.

- Additional influence of chemical effects, approximated by reduced $E_{S B E}$.

- Additional influence of roughness approximated by increased angle of incidence of projectile ions.

- Both additional effects included in simulation.

All these cases were studied as function of the $\mathrm{C}$ fraction in the incident flux to evaluate the influence of the particular factors (or their combination) on the W sputtering yield and the carbon implantation and to evaluate their influence on the simulation of the experimental results. A comparison of experimental data and the results of the simulations with the different sets of assumptions is shown in Figure 5. The transition from $\mathrm{W}$ erosion to $\mathrm{C}$ layer deposition has been found in the experiments to occur in the range $f_{C}=13-16 \%$ (see Figure $5 b)$. The experimental uncertainty in the determination of the transition point is caused by slight variations in the evolution of surface roughness for different samples.

The best fit between simulation and experiment of the two steady state parameters was obtained for the simulations including only the roughness approximation without the additional $E_{S B E}$ modification for approximation of chemical effects. Including roughness is essential for the simulation, because it characterizes the condition of the surface. Without the 
surface roughness approximation, experimental results and simulations strongly disagree for both the $\mathrm{W}$ sputtering yield and the $\mathrm{C}$ steady-state areal density. One should note further that the simulations fail to accurately reproduce the experimental $\mathrm{C}$-fraction characterising the transition from steady state $\mathrm{W}$ sputtering to continuous $\mathrm{C}$ layer growth. The default TRIDYN model results in a transition point at $f_{c}=7-8 \%$, by a factor 2 below the measured value, while inclusion of any additional assumptions shifts the transition point to $f_{c}$ values above $20 \%$. In contrast, for the case of simultaneous $3 \mathrm{keV} \mathrm{He}$ and $6 \mathrm{keV} \mathrm{C}$ ion bombardment of a W surface where chemical and roughness effects are negligible, TRIDYN is able to correctly reproduce the measured transition point [10]. Therefore, a more detailed description of surface roughness and chemical effects will be required to improve the model.

While the numerical simulation does not require chemical effects to match the experimental data and the inclusion of chemical effects into the simulations does not allow to fit the results of different experiments in a systematic way, this is still not sufficient to principally exclude the influence of chemical effects. Indeed, in the simulations, the influence of roughness and chemical effects actually appears similar: both processes result in an increase of the $\mathrm{W}$ sputtering yield and a corresponding decrease of the $\mathrm{C}$ steady state areal density. However, the experimental evidence discussed in section 3 strongly indicates that chemical effects in the given system have no significant influence on the measurements. Therefore roughness is the only factor left to explain the pronounced shift of the transition point.

\section{Conclusions}

Tungsten was bombarded with $6 \mathrm{keV} \mathrm{C}$ ions and $3 \mathrm{keV} \mathrm{D}$ ions. The sputtering of $\mathrm{W}$ and the implantation of $\mathrm{C}$ was systematically studied as a function of the $\mathrm{C}$ fraction in the incident flux at room temperature. The experiments have shown that simultaneous implantation of $\mathrm{C}$ 
and $\mathrm{D}$ in $\mathrm{W}$ does not lead to an initially expected increase of $\mathrm{D}$ retention. Moreover, the concentration of implanted $\mathrm{C}$ exceeds the concentration of retained $\mathrm{D}$ in the implantation zone by an order of magnitude. This will strongly decrease formation of hydrocarbon molecules on top of the surface preventing significant contributions to erosion of $\mathrm{C}$ by kinetically induced chemical sputtering. Comparison of TRIDYN simulations with measurements of the evolution of the surface composition can explain the observed W sputtering yield and areal density of retained $\mathrm{C}$ if one includes effects of surface roughness. While the simulations can also be matched to the experiment in a less systematic way by assuming chemical effects, the low D concentration measured in the implantation zone suggests that they do not play a significant role. Therefore, surface roughness is left as the only possible factor responsible for significant shift of the transition point from continuous $\mathrm{W}$ erosion to $\mathrm{C}$ layer growth towards higher $\mathrm{C}$ fractions in the incident flux.

However, at higher temperatures chemical effects may lead to significantly higher $\mathrm{C}$ sputtering yields, which might be balanced by a lower D retention. This issue will be addressed in future experiments. One has to note that in this case comparison of weight-loss measurements and simulations may be incorrectly interpreted due to the uncertain contribution of roughness. The detection of eroded hydrocarbon molecules and radicals in the mass spectra of sputtered particles is a suitable diagnostic for the identification of chemical erosion.

\section{Acknowledgements}

The authors would like to thank Dr. A. Gigler for AFM measurements; P. Matern, G. Matern, S. Lindig, M. Fusseder, J. Dorner for valuable technical assistance. 


\section{References}

[1] G. Janeschitz, ITER JCT and ITER HTs, J. Nucl. Mat. vol. 290-293 p.1 (2001).

[2] K. Krieger et al., J. Nucl. Mat. Vol.266 -269 p.207 (1999).

[3] A.S. Kukushkin, H.D. Pacher et al., J. Nucl. Mat. 337-339 (2005) 50.

[4] K. Schmid, K. Krieger, A. Kukushkin, A. Loarte, J. Nucl. Mat. 363-365 (2007) 674.

[5] W.Eckstein, J.Roth, Nucl. Instrum. and Methods in Phys. Res., vol.B83, p.279 (1991).

[6] I. Bizyukov, K. Krieger, N. Azarenkov, U. von Toussaint, J. Appl. Phys. 100 (2006) 113302.

[7] W. Moeller, W. Eckstein, J.P. Biersack, Comput. Phys. Commun. Vol.51 p.355 (1988).

[8] W. Eckstein, K. Krieger, J. Roth, J. Nucl. Mater. Vol.258-263 p.912-916 (1998).

[9] K.Schmid, J.Roth, J. Nucl. Mater. vol.313 -316 p.302 -310 (2003).

[10] I. Bizyukov, K. Krieger, J. Appl. Phys. 101, 104906 (2007).

[11] W. Eckstein, Computer Simulation of Ion-Solid Interactions, Springer Series in Material Science Vol. 10 _Springer, Berlin, 1991

[12] K.Schmid, J.Roth. J. Nucl. Mater. v. 302, p. 96 ( 2002)

[13] S.Y. Tong, W.N. Lennard, P.F.A. Alkemada, I.V. Mitchell, Nucl. Instrum. Methods B45 (1990) 41

[14] I. Bizyukov, K. Krieger. Rev. Sci. Instrum. 77 (2006) 043501

[15] I. Bizyukov, K. Krieger, N. Azarenkov, S. Levchuk, Ch. Linsmeier, J. Nucl. Mat. 337- 
Page 16

$339(2005) 965$.

[16] Sputtering by particle bombardment. II. Sputtering of alloys and compounds, electron and neutron sputtering, surface topography. Editored R. BEHRISCH. Topics in Applied Physics. Vol. 52. ISBN 3-540-12593-0. Berlin/Heidelberg/New York/Tokyo: SpringerVerlag 1983.

[17] W. Wang, J. Roth, S. Lindig, C. H. Wu. J. Nucl. Mater. 299 (2001) 124

[18] T. Shimada, H. Kikuchi, Y. Ueda, A. Sagara, M. Nishikawa. J. Nucl. Mater. 313-316 (2003) 204-208.

[19] Alimov VK, Ertl K, Roth J, Schmid K J. Nucl. Mater. 282 (2000) 125.

[20] J. Roth. J. Nucl. Mater. 266-269 (2001) 51-57

[21] E. Salonen, K. Nordlund, J. Keinonen and C. Wu. Phys. Rev. B, 63 (2001) 195415

[22] M. Balden, J. Roth. J. Nucl. Mater. 280 (2000) 39

[23] C. Hopf, A. von Keudell, W. Jacob. Nucl. Fusion. 42 (2002) L27

[24] W Jacob, C Hopf, M Schluter. Phys. Scr. T124 (2006) 32 


\section{List of figure captions}

Figure 1. Blister formation of the tungsten layer. (a) - optical microscopy; (b) - scanning electron microscopy of the cross-section of the sample.

Figure 2. Depth distribution of implanted D, obtained with NRA. (a) - implantation of D in pure W surface with a total fluence of $15 \times 10^{18} \mathrm{~cm}^{-2}$; (b) - simultaneous bombardment with $3 \mathrm{keV} \mathrm{D}$ and $6 \mathrm{keV} \mathrm{C}$ ions $\left(f_{C}=6 \%\right)$ with a total fluence of $4.1 \times 10^{18} \mathrm{~cm}^{-2}$.

Figure 3. Depth profile of C implanted in W calculated with TRIDYN. Projectiles are $6 \mathrm{keV} \mathrm{C}$ ions and $3 \mathrm{keV} \mathrm{D}$ ions $\left(f_{C}=6 \%\right)$, incidence angle is $38^{\circ}$. The different lines correspond to different surface binding energies of $\mathrm{C}$ atoms to $\mathrm{C}$ surface: $7.6 \mathrm{eV}$ (domination of collisional sputtering) and $3.6 \mathrm{eV}$ (approximation for chemical sputtering).

Figure 4. Implantation-sputtering curves. Each point shows areal densities of W sputtered and C implanted at the same fluence. Percentage values in the legend refer to the carbon fraction $f_{C}$. (a) - steady-state sputtering; (b) - continuous C layer growth. Parameters used in TRIDYN: $E_{S B E}=7.6 \mathrm{eV}, \alpha=38^{\circ}$.

Figure 5. Parameters of steady-state ion-surface interaction in dependence on $\mathrm{C}$ fraction in total incident flux: (a) - areal density of C implanted; (b) - sputtering yield of W. 